\title{
Prospección arqueológica: Okolhuitz, Campeche
}

\author{
Archaeological Prospecting: Okolhuitz Campeche
}

Adán Pacheco-Benítez

Resumen: El objetivo principal de este trabajo es mostrar la importancia de la prospección arqueológica como método para ir más allá del simple conocimiento de la distribución de sitios arqueológicos en la región Río Bec. De esta manera, se muestran los trabajos de prospección realizados en el sitio arqueológico de Okolhuitz. Campeche, y sus resultados, con el fin de que permitan avanzar en el conocimiento y análisis del patrón de asentamiento en la región en la que se encuentra situado.

Palabras clave: prospección arqueológica, mayas.

Abstract: The main objective of this paper is to show the importance of archaeological exploration as a method that would allow us to go beyond the mere knowledge of the distribution of archaeological sites in the Rio Bec region. In this manner, It shows the prospecting work carried out in the archaeological site of Okolhuitz. Campeche, and its results in order to make it possible to move forward with the knowledge and analysis of the settlement pattern in the region in which it is located.

Keywords: archaeological exploration, Mayas.

Adán Pacheco Benítez, arqueólogo del Centro INAH-Chiapas, México. Temas de especialización: arqueología maya. Correo electrónico: benitez_pacheco@yahoo.com.mx.
Enviado a dictamen: 21 de junio de 2013. Aprobación: 05 de agossto de 2013. Revisiones: 1. 
E estudio del patrón de asentamiento en el área maya ha concentrado un buen número de nvestigaciones; entre ellas, podemos mencionar los estudios sobre Uaxactún (Acevedo y Paz, 1991), Calakmul (Fletcher et al., 1987), Palenque (Barnhart, 2001), Chinikinhá (Liendo, 2000, 2005), Edzná (Andrews, 1984) o Caracol (Jaeger, 1991). Como vemos, se trata de trabajos llevados a cabo en sitios arqueológicos con una larga historia de investigaciones que han incluido extensas labores de recorrido y mapeo; sin embargo, sitios más pequeños o aislados no han recibido la atención necesaria para analizar dicho patrón de asentamiento. En este último caso se encuentra el sitio de Okolhuitz, objeto de estudio en este trabajo (ver mapa 1).

La prospección arqueológica ha sido escasamente valorada y juega un papel secundario en la investigación arqueológica porque la mayoría de los trabajos se han centrado en la excavación. Esta situación comenzó a cambiar, a nivel general, con la aparición de la "arqueología del paisaje" o "arqueología del territorio", en el contexto teórico de la new archaeology (Hodder y Orton, 1976). En el caso de México, son contados los proyectos que centran su atención en el conocimiento del territorio y que intentan hacer algo más que llenar huecos en la cartografía arqueológica mexicana. Sin embargo, por las causas que motivaron el recorrido y que explicaremos más adelante, podemos decir que la información presentada, junto con otras prospecciones realizadas, puede servir para plantear un proyecto de prospección adecuado de la zona que permita analizar el patrón de asentamiento y la distribución de sitios arqueológicos en un territorio más amplio, así como la recolección apropiada de materiales cerámicos.

El trabajo de prospección que permitió la recopilación de información en el sitio de Okolhuitz inició con motivo del asfaltado del tramo que une el ejido 20 de Noviembre con la carretera EscárcegaChetumal (186), municipio de Calakmul, Campeche. Los trabajos se realizaron en el año 1998 y se incluyeron dentro del Proyecto Caminos del Mundo Maya del Instituto Nacional de Antropología e Historia (INAH). Este proyecto englobaba otros recorridos, como el camino de Candelaria a El Tigre - sitio arqueológico y poblado-y el libramiento del poblado de Chiná, cerca de la capital del estado.

El objetivo del recorrido fue el de reconocer el área del sitio de Okolhuitz, que hasta ese momento había sido poco documentada. La última noticia que se tenía sobre el sitio fue la de la intervención del año 1995 (Benavides, 1997). En este recorrido se complementó la información con la que se contaba hasta entonces, ya que se reportaron tres conjuntos más que, como se presentará más adelante, son en realidad parte del Okolhuitz reportado por Ruppert y Denison (1943).

Cabe señalar que han pasado varios años desde que realizamos el mencionado recorrido, pero por causas ajenas no habíamos podido publicar los resultados. Sin embargo, consideramos que la información recopilada durante este trabajo puede ser de utilidad. Si bien es cierto que hay investigadores que siguen recorriendo el área actualmente, el presente trabajo sólo trata de contribuir al mejor entendimiento del territorio, particularmente del sitio de Okolhuitz.

\section{Antecedentes}

Los trabajos de prospección en el ramal de 20 de Noviembre con la carretera Escárcega-Chetumal tuvieron una extensión de 5200 metros y se iniciaron en el sitio de Okolhuitz, que se localiza en el kilómetro 3.48 del ramal que parte de la carretera 186, a 488 metros al oriente del mismo. El sitio ya había sido reportado por los arqueólogos Karl Ruppert y John H. Denison (1943), del Instituto Carnegie, de Washington.

El lugar fue llamado Okolhuitz, que de hecho es una corrupción del maya yucateco holhuitz "arriba de la colina", o bien, okolhuitz, "entrar a la colina" (Benavides, 1997).

En la publicación de Ruppert y Denison (1943: 8384, plano 71) sobre el recorrido de reconocimiento que realizaron por Campeche, Quintana Roo y El Petén describen sólo seis estructuras - las ahora numeradas del 1 al 6-, que tal vez se consideraban las más importantes en su momento. Posteriormente, en los inicios de la década de los noventa, el sitio fue visitado 
nuevamente por el arqueólogo Ricardo Bueno Cano. Cuatro años más tarde, en 1994, es "redescubierto" y fotografiado por Nicholas Hellmuth y Andrea David. Finalmente, en 1995 el sitio es reconocido por Antonio Benavides con el Proyecto de Mantenimiento a Zonas No Abiertas al Público (MANZANA). En el proyecto se trabajaron las estructuras 1, 2, 7, 8, 14 y 15, en las que se realizaron labores de consolidación de paramentos o bóvedas, además de elaborar un croquis: "No incluye muchos montículos y plataformas observadas en los alrededores de los inmuebles registrados. La estela 1 es lisa y la número 2 podría haber contado con alguna representación" (Benavides, 1997: 33). El croquis muestra solamente 31 estructuras del sitio e incluye las reportadas en 1943 (ver mapa 2).

Comopodemosver, el total de estructuras registradas y topografiadas es de 107, las cuales no habían sido reportadas con anterioridad. A éstas debemos añadir las 31 que sí habían sido registradas con anterioridad, las cuales reflejan un total de 138 estructuras mapeadas desde Rupert y Denison hasta el momento de nuestro recorrido.

\section{Los trabajos}

Como expresé en el apartado anterior, se tomó como referencia el sitio de Okolhuitz, lo cual fue de gran utilidad debido a que al sureste de la Estructura l, a unos 300 metros, se localizó la continuación del mismo. Esta parte recién registrada la denominamos Okolhuitz II para dejar registro de dicho trabajo (ver mapa 3).

Otro conjunto que se localizó fue llamado La Ciudadela. Se denominó así debido a que su área monumental se encuentra rodeada por una serie de palacios que delimitan específicamente dicha área. El nuevo asentamiento se localiza al sureste de la Estructura 1 de Okolhuitz, a poco más de un kilómetro de distancia. El área estimada donde se concentra la parte monumental es de 265 metros norte-sur y de 185 metros este-oeste. Se compone de una gran plataforma sobre la que se localizan todos los edificios, que a su vez se agrupan en diferentes niveles de plataformas y pequeñas plazas, hasta llegar a una plataforma que soporta la Estructura l del sitio, la cual se encuentra aislada. Al poniente y en un nivel inferior se observa un Juego de Pelota, con dos oquedades de saqueo en la estructura este, en su costado oriente. El área monumental está conformada por 22 estructuras incluyendo las dos estructuras del Juego de Pelotaen las que se observan plataformas, basamentos con edificio o templo en su parte superior y edificios con dos o cuatro cuartos. Los cuartos centrales se comunican entre sí, mientras que los laterales son independientes y cuentan con sus respectivos accesos.

Cruzando el trazo de la carretera, al poniente de Okolhuitz, se localizó otro asentamiento al parecer más modesto, a unos 600 metros del primero. Éste se encuentra en la parte superior de una colina que no rebasa los 70 metros de altura. Lo que se pudo observar es la presencia de plataformas y montículos que no rebasan los tres metros de altura. Una vez se llega a la parte más alta, se pueden ver tres edificios que forman un patio abierto, puesto que en su parte poniente no se encontró estructura alguna. En el edificio que se localiza en la parte oriente del conjunto se conserva aún en pie, parte del muro central y restos de una bóveda conservada gracias a las raíces de los árboles que han crecido sobre la estructura, pero que en el futuro terminarán por destruir los restos de bóveda y paramentos.

Asimismo, se abrieron brechas en ambos costados de la carretera. En ellas se halló una constante de montículos de menor tamaño que no rebasan los cinco metros de altura, por lo que podemos suponer que se trata de unidades habitacionales y pequeñas plataformas. Cabe destacar que un elemento reiterativo en esta área es la aparición de albarradas o largas plataformas que asimétricamente sustentan un pequeño altar. Su altura no sobrepasa el metro y medio.

En cuanto a la funcionalidad de las albarradas, proponemos tres hipótesis: la primera sería que éstas sirvieran como limitante de solares familiares, incluidas dos o tres unidades habitacionales.

La segunda es que las albarradas desempeñaran la función de muros divisorios entre parcelas de cultivo. Este mismo patrón de albarradas ya ha sido reportado 
en Nicolás Bravo, Quintana Roo, en partes de la costa del mismo Quintana Roo y en la parte norte de Yucatán (comunicación personal de Enrique Nalda, 1998). Por ello, tal vez éstas son las primeras en las que se reporta su localización con precisión en el sur de Campeche, debido a que en la literatura existente para el área de Río Bec no se ha especificado con exactitud la localización de las albarradas de Becán y del este de Xpuhil (Thomas, 1981). Dichas albarradas no tienen una orientación definida ya que la mayoría de las veces siguen el contorno del terreno irregular de las pequeñas colinas en las que se asientan dichas estructuras.

La tercera hipótesis sería que algunas de estas albarradas no funcionaron como linderos de unidades familiares, sino que sirvieron como canales o ductos para la conducción del agua. El motivo que nos llevó a pensar esto fue que constructivamente presentan un vacío de forma rectangular. Además, se pudo observar una serie de piedras labradas en forma de tapas de bóveda, las cuales cerraban la parte superior de estos conductos. En cuanto a su profundidad, no pudo ser determinada puesto que no se realizó ningún tipo de intervención.

Recolectar materiales culturales, realizar pozos de sondeo, o bien practicar excavaciones intensivas o extensivas, habría sido de gran utilidad para definir con mayor certeza la función y uso de las albarradas.

En otra brecha se localizó el asentamiento al que se denominó Las Albarradas, debido a que tiene una serie de muros no mayores de 1.50 metros de altura y aproximadamente dos metros de anchura. Estas albarradas muestran una forma serpenteante y alcanzan en ocasiones 50 metros de largo.

Las albarradas se interconectan y al mismo tiempo se observan pequeños vanos que dan la impresión de ser entradas a espacios delimitados por largos muros. Otro aspecto que se pudo observar es que en algunas partes hay muros de contención para la nivelación del terreno, por lo que forman terrazas de baja altura sobre las que se desplantan largos muros, así como pequeñas estructuras que no rebasan los tres metros de altura. Estas estructuras parecen ser unidades habitacionales, y una, dos o tres de ellas pueden estar en estos espacios limitados por las albarradas.
Hasta el momento, este tipo de arquitectura está presente en los sitios que se mapearon -Okolhuitz I y II, La Ciudadela y Las Albarradas-. Este patrón de asentamiento concentrado y constante se mantiene a lo largo de los cinco kilómetros de carretera recorridos. Las brechas inspeccionadas tenían un largo de 500 metros a partir del centro de la carretera, por unos cinco metros de ancho en forma transversal a ella, por lo que se abarcó un promedio de un kilómetro de distancia. Podemos afirmar que en las partes bajas sólo se encuentran las albarradas, unidades habitacionales y áreas de concentración de desecho de materiales, en este caso sílex únicamente. Por otra parte, los edificios suntuarios o administrativos se localizan en las partes altas de las colinas, de tal manera que se repite en estos la constante de albarradas o canales. En algunas ocasiones se encuentran edificios aislados en las partes bajas, los cuales se anegan en tiempos de lluvia.

\section{Descripción de los conjuntos}

Al término de los trabajos de prospección y topografía, podemos anexar al sitio de Okolhuitz los siguientes tres: Okolhuitz II, La Ciudadela y Las Albarradas. El número de estructuras registrado en estos tres nuevos sitios es el siguiente:

- Okolhuitz II: 45 estructuras.

- La Ciudadela: 22 estructuras.

- Las Albarradas: 40 estructuras.

\section{Okolhuitz}

Los dos croquis con los que contaba el sitio hasta el momento eran los de Ruppert y Denison (1943: 83-83, plano 71 y figs. 103-104) y el de Benavides (1995), los cuales contemplaban el Okolhuitz conocido hasta entonces. Éstos fueron completados con el presente trabajo.

Entre ambos croquis sólo aparece registrado un total de 31 edificios. En la actualidad, esta cifra se vio incrementada con la localización de otras estructuras. El recorrido realizado entre los meses de agosto y octubre de 1998 dio como resultado la aportación de 45 
estructuras más. La disposición de las mismas muestra un patrón de plazas cerradas o abiertas limitadas por dos o tres edificios que, en algunos casos, se desplantan sobre nivelaciones artificiales - plataformas- o bien directamente sobre el nivel del terreno natural. Asimismo, aparecen edificios aislados, de dimensiones similares, rodeados por montículos de baja altura.

Porellopodemos suponerque Okolhuitzcomprendía una superficie mayor a cinco kilómetros cuadrados. Esto lo observamos en los recorridos realizados en los alrededores del asentamiento conocido hasta el momento, dado que éste se extendía hacia los cuatro puntos cardinales, formando así un patrón continuo de estructuras.

El sitio también fue mencionado por Paul Gendrop, aunque sólo lo analiza desde el punto de vista arquitectónico. De ello nos dice:

Es muy probable que durante esos mismos años 550 y 600 Clásico Medio de transición hacia el pleno florecimiento Clásico, muchos de los otros sitios de la región se hayan convertido a su vez en centros de cierta importancia, como pudo ser el caso, al norte de Becan, de Péchal y Peor Es Nada, de Xpuhil y Okolhuitz al este, y al sur Chicanná y Hormiguero así como del conjunto de grupos que integraban el sitio de Río Bec propiamente dicho. [...] No resultaría sorprendente, por cierto, que datara de esta época el delicado Edificio I de Okolhuitz, cuyos estrechos aposentos - junto con la distribución de su planta, la inclinación de sus techos y otros rasgos atípicosparecerían más bien relacionarse con las tradiciones del Peten (Gendrop 1983: 29).

\section{Okolhuitz II}

El recorrido realizado en este sector de Okolhuitz se inició en una brecha que partió de la Estructura ly, a unos 300 metros al sureste, localizamos una serie de estructuras que rebasan los diez metros de altura y que se hallan dispuestas en la parte elevada de varias colinas que no rebasan los 80 metros de altura. Al parecer, parte de estas estructuras presentan muros de contención para así crear terrazas de nivelación constituidas por sillares burdamente trabajados y con presencia muy marcada de la utilización de cuñas a la manera de la arquitectura de El Peten. De igual forma, las albarradas presentan este mismo tipo de trabajo y siguen el contorno irregular del terreno, por lo que no tienen una orientación específica (ver mapa 3).

Como se mencionó anteriormente, se mapearon un total de 45 estructuras en el área recorrida de Okolhuitz. Los edificios que componen el sitio están dispuestos en plazas abiertas que son sustentadas por plataformas artificiales, cuya altura varía de acuerdo con el desnivel del terreno. Como se pudo observar, algunos edificios se componen de cuartos dobles - anterior y posteriorcomunicados entre sí por un vano central.

Otro caso son las estructuras compuestas por cuatro aposentos; los centrales son iguales a los expuestos en el caso anterior, y los dos de los extremos son independientes de los centrales.

En algunas circunstancias se localizaron edificios que, aparentemente, sólo comparten el espacio de la plataforma con unidades habitacionales. En otras partes del conjunto se observan únicamente unidades habitacionales o bien plataformas de baja altura.

En cuanto a restos arquitectónicos, se observaron los siguientes: el muro intermedio con restos de bóveda y los edificios que contienen cuartos dobles, anteriores y posteriores. En otros casos se apreciaron partes de los muros exteriores que a simple vista no conservan ningún elemento decorativo en sus muros o frisos. Como únicos ejemplos de arquitectura y decoración en pie, se observaron los edificios 1, 2 y 15.

Del recorrido realizado podemos afirmar que Okolhuitz II rodea prácticamente a Okolhuitz, y que es un asentamiento con una gran concentración de edificios, plazas, patios, unidades habitacionales, albarradas, plataformas y canteras para la extracción de piedra. De igual manera, algunos edificios no son los "típicamente Río Bec" — torres con escalinatas impracticables rematadas con un templo simulado, esquinas redondeadas y fachadas zoomorfas, entre otras características - ya que en varios no se contemplan los rasgos distintivos del mencionado estilo arquitectónico, 
sino que muestran una arquitectura más temprana que correspondería al tipo Petén. Lo anterior se observa claramente por el tipo de trabajo plasmado en los sillares, ya que en el estilo Petén éstos se manufacturaron toscamente y muestran un uso frecuente de cuñas y abundante utilización de argamasa, además de no presentar esquinas o aristas bien definidas.

En otros casos se observa una clara arquitectura riobequeña bien definida, caracterizada por los sillares que componen los paramentos, los cuales ya reflejan una técnica de labrado de piedra y muestran un acabado bien definido en sus esquinas, por lo que el uso de cuñas es ocasional. Además, los bloques en su mayoría tienden a ser cuadrados $y$, si llevan argamasa entre piedra y piedra, es mínima.

De esta manera, Okolhuitz y Okolhuitz II forman un patrón de asentamiento del tipo concentrado. Es decir, hay una constante entre los grupos residenciales, administrativos o religiosos debido a que no se encuentran aislados unos de otros; por el contrario, en sus alrededores se observan pequeños montículos a manera de unidades habitacionales donde seguramente se albergaba la gente que atendía a la élite del sitio.

Hasta el momento no se han localizado juegos de pelota en ninguno de los dos asentamientos. El único observado en todo el recorrido se encuentra en el sitio de La Ciudadela, a poco más de un kilómetro de distancia.

\section{LaCiudadela}

Se localiza al noroeste de la Estructura 1 de Okolhuitz. Hasta el momento se han localizado 22 estructuras, como edificios de dos, cuatro y posiblemente seis o más aposentos. La forma del asentamiento es rectangular y está ubicado en un eje norte-sur de 288 metros en su parte más prolongada, mientras que el eje este-oeste no rebasa los 185 metros de ancho. La mayor concentración de edificios se localiza en el eje norte-sur (ver mapa 4).

La Estructura 1 se desplanta sobre uno de los niveles más altos del sitio, de tal manera que queda sola sobre este desnivel. Justo al frente del Edificio ly al centro del sitio, en un nivel más bajo, se localiza el Juego de Pelota que se mencionó líneas arriba. Éste es del tipo abierto, por lo que tal vez sea tardía su construcción. Al oeste del Juego de Pelota se encuentra otro palacio — siendo éste el más largo del sitio-, en cuya parte posterior se observa parte de su núcleo debido a que presenta huellas profusas de saqueo.

En el costado sur del Edifico l se encuentra la otra concentración de estructuras que tienden a formar plazas delimitadas solamente por dos estructuras, de tal manera que forman plazas abiertas. En esta parte del sitio es donde se observa un mayor desnivel en el terreno debido a que las estructuras ahí construidas fueron desplantadas sobre plataformas con diferentes alturas.

En la parte suroeste del conjunto solamente se observa una serie de plataformas de menor tamaño, a diferencia del resto del asentamiento.

El costado sur del conjunto se encuentra delimitado por un edificio posiblemente constituido sólo por dos cuartos y una plataforma de unos 40 metros de largo. Fuera de este costado, se encuentra aislada otra plataforma de unos 20 metros de largo por unos seis de ancho y 1.50 metros de altura.

El conjunto este, delimitado al norte por una serie de edificios en forma de palacio, se puede observar claramente a partir del costado norte de la Estructura 1. El total de estos edificios en forma de palacio es de diez. El conjunto este limita al norte con tres estructuras, tres más al este, otras tres al sur y una más al oeste, esta última la más larga del conjunto norte. Éste constituye prácticamente el único conjunto perfectamente delimitado por sus cuatro costados.

\section{Las Albarradas}

Este conjunto se encuentra en el kilómetro 0.84 y se localizó en la brecha 3. El asentamiento consiste en una serie de albarradas que miden más de 50 metros de largo por una altura de 1.50 metros en promedio; su orientación es indefinida ya que estas albarradas son de forma serpenteante y en ellas se observan pequeños vanos a manera de accesos. Asimismo, puede verse que se unen unas con otras formando patios donde se encuentran de una a tres unidades habitacionales que no rebasan los 2.5 metros de altura, a excepción de dos o 
tres edificios que posiblemente fueron utilizados como templos. Los patios no cuentan con ninguna estructura o construcción. En términos generales, puede decirse que aparentemente no hay un orden en la distribución de las unidades habitacionales ni en la forma de los patios que forman las albarradas, por lo que el patrón de distribución es irregular. En las estructuras del conjunto no se observó la presencia de plazas bien definidas.

\section{Consideraciones generales: Okolhuitz, La Ciudadela y las Albarradas}

Resumiendo brevementelodescrito, podemos proponer, primero, que ambos Okolhuitz en realidad son uno debido a su cercanía. Además, los recorridos realizados anteriormente por otros investigadores no fueron más allá de las estructuras la la 31 (ver croquis de Benavides, 1995; Ruppert y Denison, 1943). Prácticamente, Okolhuitz II rodea al Okolhuitz reportado por Ruppert y Denison. Además, se pudo apreciar una constante en los estilos arquitectónicos, de manera que en general podría proponerse como un sitio transicional entre los estilos de Petén y de Río Bec. Si esto es así, podríamos confirmar que Okolhuitz contempla una larga secuencia constructiva y podríamos plantear sus inicios, cuando menos, en el Preclásico Terminal. Los rasgos estilísticos no sólo muestran meramente una tendencia al estilo Río Bec, como sería el caso de Río Bec, sitio ubicado a 16 kilómetros de Okolhuitz, Hormiguero, a 12 kilómetros, o Ramonal, a tres kilómetros. Estos últimos sí atestiguan una arquitectura totalmente Río Bec. No es difícil pensar que en etapas constructivas previas estos sitios también guarden reminiscencias del estilo Petén, que yacen ocultas bajo los edificios.

En segundo término, sucede lo mismo con el sitio denominado La Ciudadela, el cual se halla a un kilómetro de distancia de Okolhuitz. Entre estos conjuntos hay una constante: plataformas de baja altura, unidades habitacionales, edificios que se encuentran aislados, las canteras de extracción de piedra, ya sea para el núcleo del edificio o para la elaboración de los sillares de revestimiento, y se siguen presentando las albarradas que delimitan los solares familiares o las áreas de cultivo. Por ello podemos pensar que, debido a una gran densidad demográfica o por la disputa de tierras a nivel interno, se vieron en la necesidad de delimitar los espacios de las unidades familiares.

La Ciudadela también podría ser parte de Okolhuitz y funciona como una extensión de este sitio, lo cual se puede afirmar debido a que la mayor concentración de estructuras monumentales, tanto en volumen como en número, se da en Okolhuitz. Por otra parte, el sitio de La Ciudadela tiene una mayor concentración de edificios tipo palacio, donde posiblemente moraba parte de la clase gobernante de Okolhuitz.

Las Albarradas guardan más semejanza con Okolhuitz que con La Ciudadela. Esto se debe a que en ambos sitios se encuentran terrazas de nivelación que no sobrepasan los 1.5 metros de altura y son constantes las albarradas.

El resto del área recorrida sólo nos proporcionó estructuras aisladas o bien pequeños conjuntos de dos o tres edificios, así como un sinfín de unidades habitacionales que siempre están limitadas por albarradas. Dentro de esta área se observaron lo que pensamos que eran áreas de actividad debido a la gran concentración de materiales de desecho, en este caso sílex, aunque no se encontró obsidiana.

\section{Conclusiones}

En los poco más de tres meses de duración del recorrido de superficie entre la carretera Escárcega-Chetumal y el ejido 20 de Noviembre, hemos podido verificar lo siguiente:

- En primer término, Okolhuitz se extiende más de lo que se había pensado hasta el momento y posiblemente aún más de lo recorrido por nosotros, debido a la continuidad que presentan las estructuras entre sí y a que no se pudo distinguir separación alguna que denotara otro posible sitio independiente en los cinco kilómetros cuadrados que hemos presentado como tentativos para Okolhuitz. 
- En segundo término, La Ciudadela se encuentra cerca de Okolhuitz, ya que apenas hay un kilómetro de distancia entre ambos sitios. Por ello, nos inclinamos a pensar que este asentamiento no es más que una extensión del primero.

- Y en tercer lugar tenemos el sitio Las Albarradas, que dista unos tres kilómetros de Okolhuitz. Si bien este sitio se presenta, hasta el momento, como el más distante, no cuenta con arquitectura monumental que pueda indicar que éste sea un sitio independiente del primero debido a que su arquitectura es del tipo de unidades habitacionales y con delimitación entre los posibles solares por medio de albarradas.

En el resto del recorrido se pudieron observar únicamente albarradas, unidades habitacionales y edificios que pudieron haber tenido uso religioso o administrativo. Estos elementos se presentan aislados de edificios de dimensiones iguales.

Otro patrón observado fue el de dos a tres edificios que forman plazas abiertas en uno o dos de sus costados, los cuales se encuentran sobre algunas de las colinas, rodeados por montículos de menor tamaño y volumen.

Por lo presentado anteriormente puede pensarse que Okolhuitz funcionó como una ciudad con poder en un amplio sector del área recorrida por nosotros. Seguramente contó con pequeñas comunidades que le aportaban mano de obra para la construcción de los edificios públicos o materia prima, o que producían alimentos.

Todo lo anterior sólo pudo ser realizado con la existencia de clases antagónicas entre sí. Por un lado, la gente que detentaba el poder, la clase gobernante, entre ellos políticos, militares, comerciantes y artesanos especializados; y, por otro lado, la fuerza productiva, la gente productora de los excedentes agrícolas, de aportación de mano de obra para la realización de edificios o plazas.

Esimportanteaclararquelaprospección arquitectónica, es decir, la observación directa en superficie de las características del construido, tanto las espaciales como las relativas los elementos componentes un "objeto arquitectónico", se considera fundamental para el entendimiento del sitio y de su cronología, por lo que se ha desarrollado una metodología de soporte a la observación directa de la realidad. Sin embargo esta debe de ser afinada en el transcurso de la próxima temporada de campo, ya que su formulación surge de trabajos precedentes realizados en otros contextos arqueológicos, y se ha modificado de acuerdo al caso específico de estudio (Liendo, 2000: 24).

Creemos que con las tres hipótesis arriba presentadas se podrá conocer más acerca del sitio de Okolhuitz, por ejemplo, la totalidad del conjunto - en la medida de lo posible-, así como, ralamente, la definición de los espacios públicos, administrativos, sus áreas de unidades habitacionales, áreas de actividad, etcétera, y con ello se entenderá mejor la funcionalidad del sitio.

Como se mencionó en un inicio, estos resultados fueron posibles por el pavimentado entre la carretera Escárcega-Chetumal y el entronque al ejido 20 de Noviembre, y se presentan como un preliminar del alcance que pueda tener un reconocimiento del área, pero ya con un proyecto específico y donde se planeen varias temporadas en campo, aunado a la recolección de materiales en superficie o en los pozos que se encuentran en diferentes parte del sitio. Todo ello proporcionaría mayor definición sobre la temporalidad de ocupación de Okolhuitz.

Sólo en parte podemos entender la presencia masiva de edificios, plataformas, arquitectura monumental y, sobre todo, esas albarradas que se observan por todas partes del área. Cabe recordar que sólo se realizó la prospección, por lo que lo presentado aquí puede ser susceptible de modificaciones posteriores.

\section{Referencias bibliográficas}

Acevedo, Renaldoy Ana María Paz Bone (1991), “Elpatrón de asentamiento de Uaxactun en el Clásico Tardío", en J.P. Laporte, S. Villagrán, H. Escobedo, D. de 
González y J. Valdés (eds.), II Simposio de Investigaciones Arqueológicas en Guatemala, 1988, Guatemala: Museo Nacional de Arqueología y Etnología, pp.157-162.

Andrews, George F. (1984), Edzná, Campeche, México. Settlement Patterns and Monumental Architecture, Eugene: University of Oregon.

Barnhart, E. L. (2001), The Palenque Mapping Project: Settlement and Urbanism at an Ancient Maya City, (tesis de doctorado) Universidad de Texas, Austin.

Benavides, Antonio (1995), "Okolhuitz, Campeche, 1995 Field Season”, en Mexicon, vol. XIX, Alemania, pp. 33-46.

Fletcher, Laraine A., Jacinto May H., Lynda Florey F. y William J. Folan (1987), Un análisis estadístico preliminar del patrón de asentamiento de Calakmul, Campeche: Centro de Investigaciones Históricas y Sociales-Universidad Autónoma del Sudeste.

Gendrop, Paul (1983), Los estilos Río Bec, Chenes y Puuc en la arquitectura maya, México: División de Estudios de Posgrado-Facultad de Arquitectura-Universidad Nacional Autónoma de México.

Goñi, Guillermo A. (1993), Solares prehispánicos en la Península de Yucatán (tesis inédita de licenciatura), ENAH, México.
Hodder, Ian y Clive Orton (1979), "Spatial Analysis in Archaeology", en New Studies in Archaeology, vol. 1, Cambridge: Cambridge University Press.

Liendo Stuardo, Rodrigo Rubén (2000), "Reyes y campesinos: datos e inferencias acerca de la población rural de Palenque", en Arqueología Mexicana, vol. III, núm. 45, septiembre-octubre, México: Editorial Raíces, pp. 34-37.

Liendo Stuardo, Rodrigo Rubén (2005), "Estrategias de dominio político regional en el reino de B'aak", en Mayab, núm. 18, España: Sociedad Española de Estudios Mayas, pp. 69-75.

Jaeger, Susan (1991), Settlement Pattern Research at Caracol, Belize: The Social Organization in a Classic Maya City, (tesis de doctorado), Department of AnthropologySouthern Methodist University, Dallas.

Ruppert, Karl y John Denison (1943), Archaeological Reconnaissance in Campeche, Quintana Roo and Petén, Washington: Carnegie Institution of Washington.

Thomas, Prentice (1981), Prehistoric Maya Settlement Patterns at Becan. Campeche, México. Nueva Orleans: Middle American Research Institute, Tulane University. 
Mapa 1. Plano general de la región del río Bec en la que se señala la situación de Okolhuiz

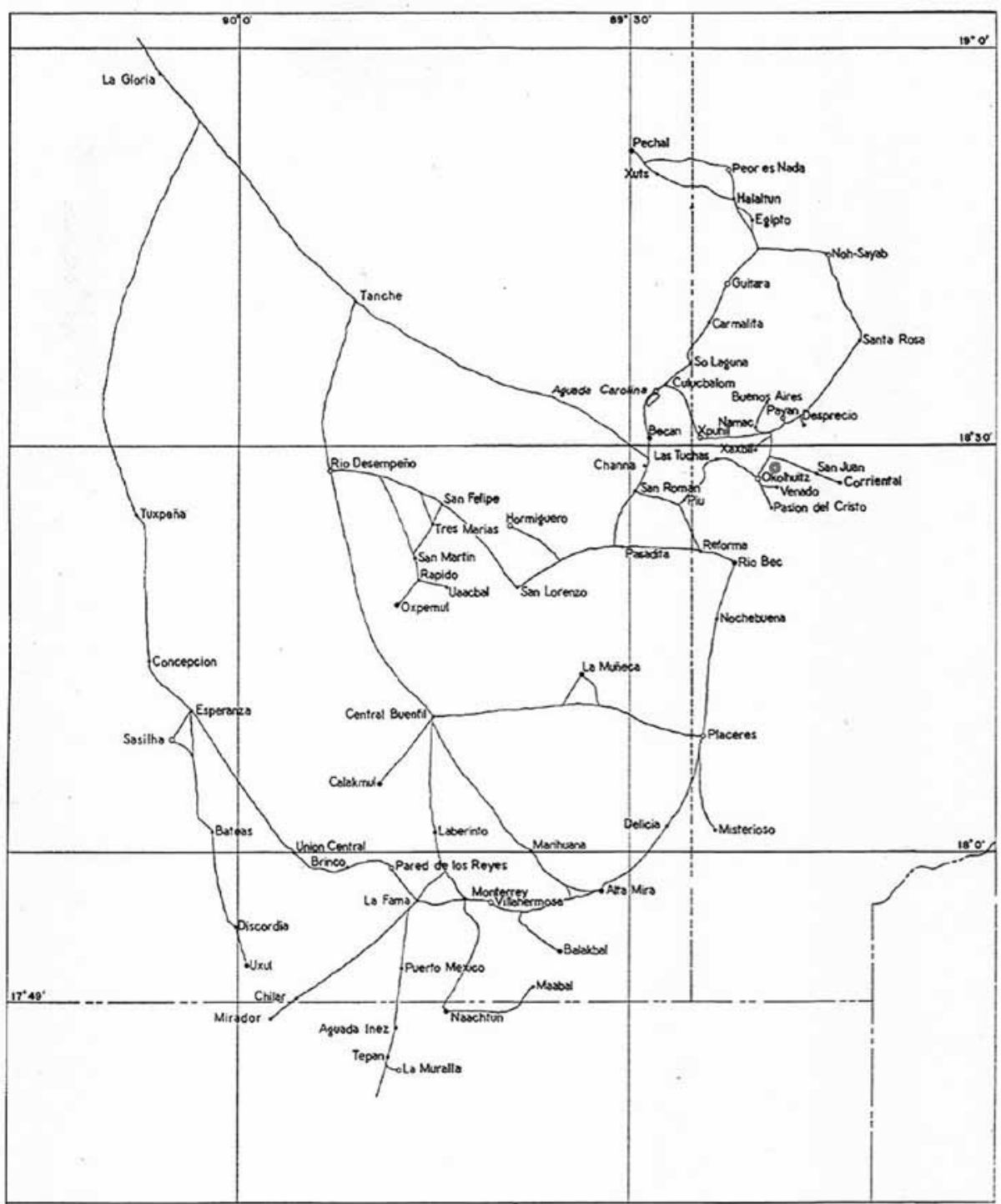

Fuente: Ruppert y Denison (1943). 


\section{Mapa 2. Plano del área de Okolhuitz}

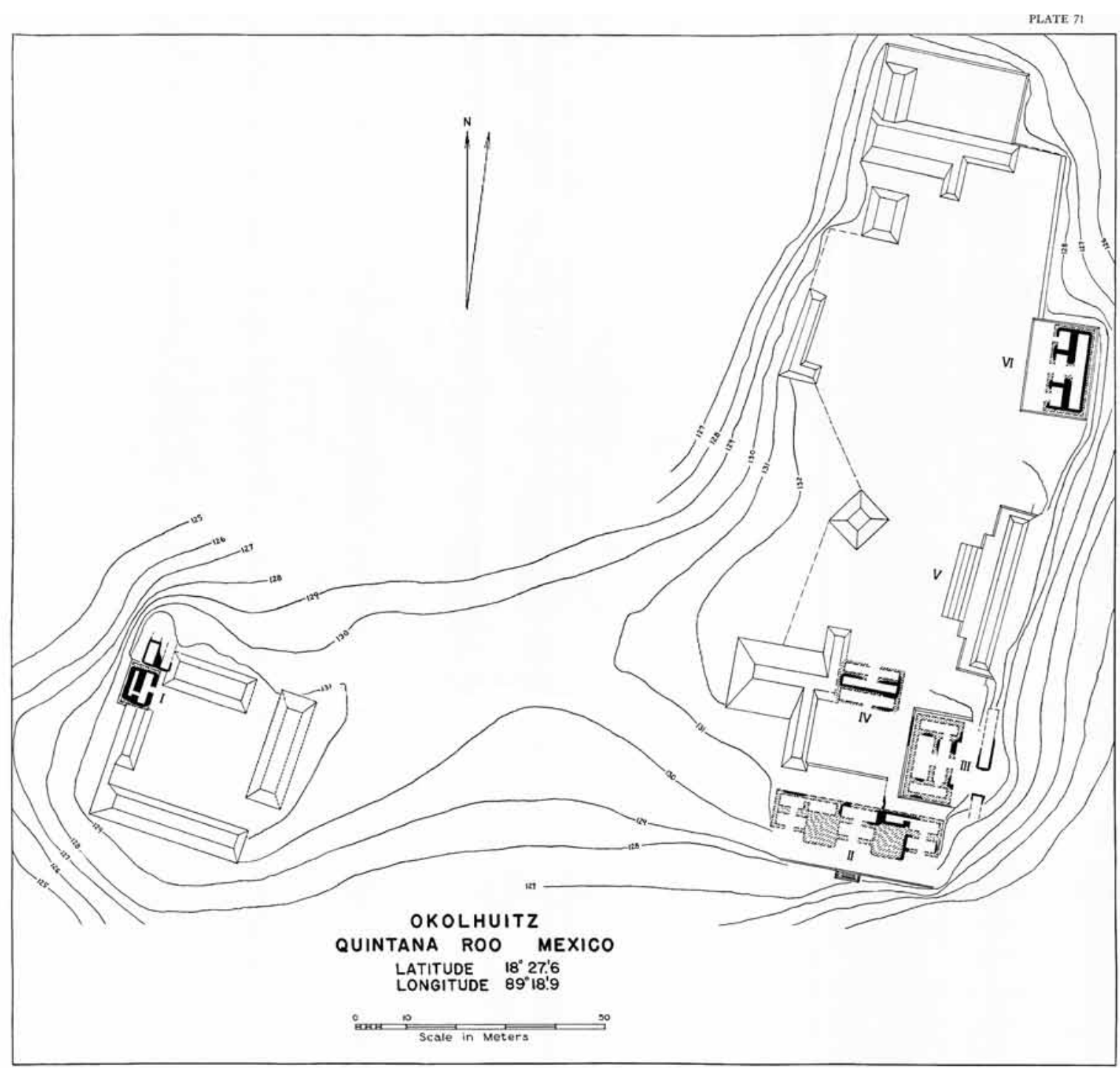

Fuente: Rupper y Denison (1943: 83-84, plano 71). 


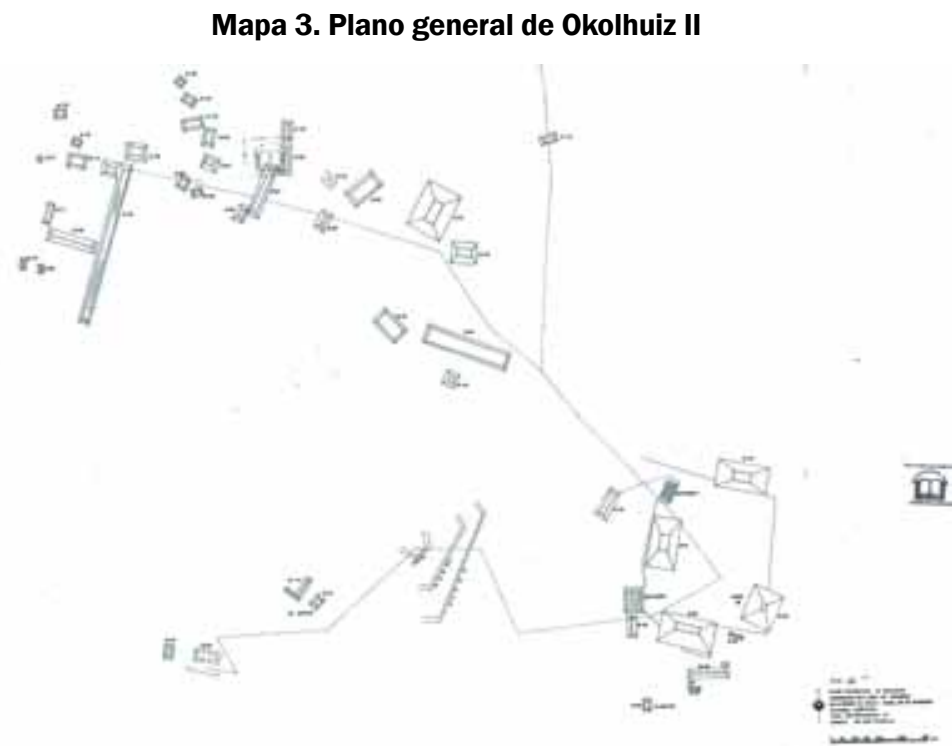

Fuente: INAH-Campeche.

Mapa 4. Plano general de la Ciudadela

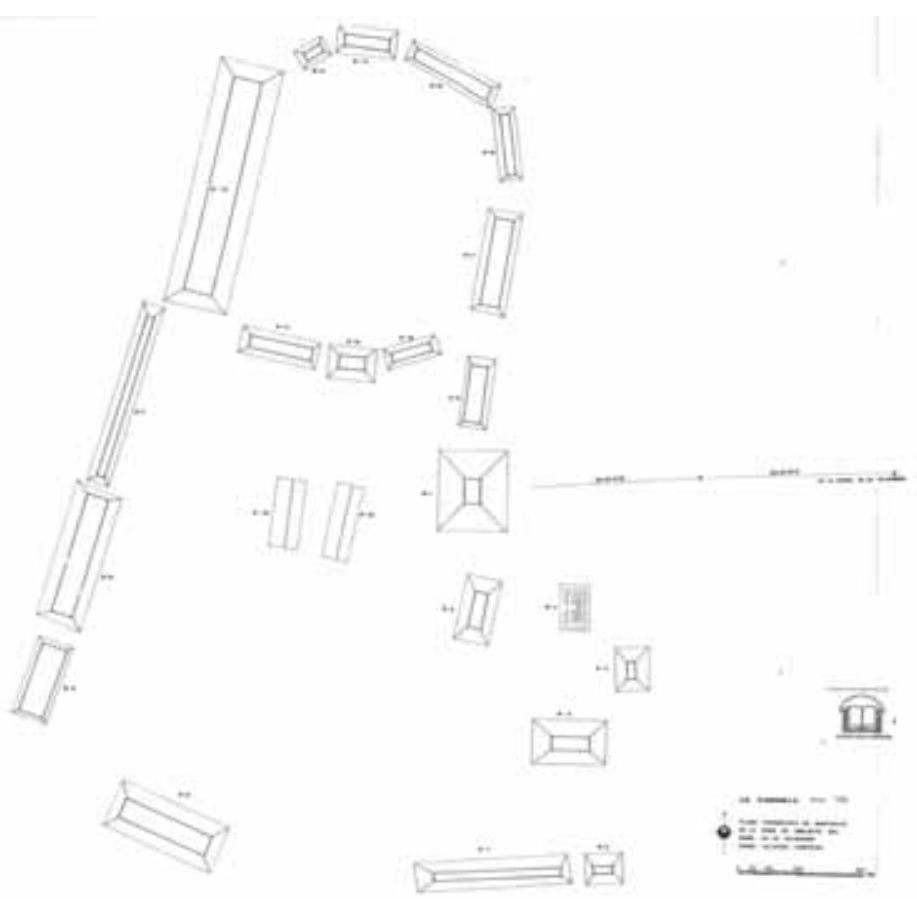

Fuente: INAH-Campeche. 